Mathematical Modelling and Analysis

Volume 20 Number 3, May 2015, 422-442

http://dx.doi.org/10.3846/13926292.2015.1050709

(c) Vilnius Gediminas Technical University, 2015
Publisher: Taylor\&Francis and VGTU

http://www.tandfonline.com/TMMA

ISSN: $1392-6292$

eISSN: $1648-3510$

\title{
Direct and Inverse Acoustic Scattering by a Combined Scatterer*
}

\author{
Jing $\operatorname{Jin}^{a}$, Jun $\mathrm{Guo}^{b}$ and Mingjian Cai ${ }^{b}$ \\ ${ }^{a}$ School of Mathematics and Statistics, Central China Normal University \\ 430079 Wuhan, China \\ ${ }^{b}$ School of Mathematics and Statistics, South-Central University For \\ Nationalities \\ 430074 Wuhan, China \\ E-mail(corresp.): hssxgj@126.com \\ E-mail: jinjing@mails.ccnu.edu.cn \\ E-mail: 38283470@qq.com
}

Received December 15, 2014; revised May 3, 2015; published online May 15, 2015

\begin{abstract}
This paper is concerned with the scattering problem of time-harmonic acoustic plane waves by a union of a crack and a penetrable inhomogeneous medium with compact support. The well-posedness of the direct problem is established by the variational method. An uniqueness result for the inverse problem is proved, that is, both the crack and the inhomogeneous medium can be uniquely determined by a knowledge of the far-field pattern for incident plane waves. The linear sampling method is employed to recover the location and shape of the combined scatterer. It is worth noting that we make the first step on reconstructing a mixed-type scatterer of a crack and an inhomogeneous medium by the linear sampling method.
\end{abstract}

Keywords: combined scatterer, inverse scattering problem, uniqueness, the linear sampling method.

AMS Subject Classification: $35 \mathrm{C} 15 ; 35 \mathrm{Q} 60 ; 78 \mathrm{~A} 45$.

\section{Introduction}

The problem that we are dealing with in this paper is the scattering of timeharmonic acoustic plane waves by a mixed-type scatterer which is given as the union of an open arc and a penetrable inhomogeneous medium with compact support. We set impedance boundary condition on one side of the arc with a constant surface impedance $\lambda$ and assume that the refractive index of the inhomogeneous medium is $n$. This model corresponds to some practical applications such as biomedical imaging, non-destructive testing and geophysical

* This research is supported by National Natural Science Foundation of Peoples Republic of China, No. 11171127 and No. 11401241. This research is also supported by the Fundamental Research Funds for the Central Universities, No. CZQ15020. 
explorations. Assuming that the electric field is polarized in the TM mode, this leads to a mixed boundary value problem for the Helmholtz equation defined in the exterior of the crack with a refractive index due to the inhomogeneous medium.

To give a precise description of the problem, we assume that the inhomogeneous region is contained in a ball $B$, the refractive index is assumed $n=1$ in $R^{3} \backslash(B \cup \bar{\Gamma})$. Let $q:=n-1$ denote the 'contrast function', then $D:=\left\{x \in R^{3} \backslash \bar{\Gamma}: q \neq 0\right\}$ is a bounded domain and can be used to denote the compact support of the penetrable inhomogeneous medium. We suppose that the crack $\Gamma$ can be extended to a $C^{2}$ smooth, simply connected and closed curve $\partial \Omega$ enclosing a bounded domain $\Omega$ such that the normal vector $\nu$ on $\Gamma$ coincides with the outward normal vector on $\partial \Omega$ which we again denote by $\nu$. Let $\Lambda$ denote the part of the boundary $\partial \Omega \backslash \bar{\Gamma}$. The bounded domain $D$ and $\Omega$ are disjoint. Let $k>0$ be the wave number and $u^{i}(x, d)=e^{i k x \cdot d}, x \in R^{3}$, be the incident plane wave with direction $d \in S$. Here, $S$ denotes the unit sphere in $R^{3}$.

The propagation of time-harmonic plane waves by a crack and a penetrable inhomogeneous medium is modeled by the Helmholtz equation with suitable boundary conditions:

$$
\begin{cases}\Delta u+k^{2} n u=0 & \text { in } R^{3} \backslash \bar{\Gamma} \\ u_{+}=0 & \text { on } \Gamma \\ \frac{\partial u_{-}}{\partial \nu}+i \lambda u_{-}=0 & \text { on } \Gamma\end{cases}
$$

where the surface impedance is $\lambda<0, u_{ \pm}(x)=\lim _{h \rightarrow 0} u(x \pm h \nu)$ for $x \in \Gamma$, and $\frac{\partial u_{ \pm}}{\partial \nu}=\lim _{h \rightarrow 0} \nu \cdot \nabla u(x \pm h \nu)$ for $x \in \Gamma$. The total field $u=u^{s}+u^{i}$ is decomposed into the given incident field $u^{i}=e^{i k x \cdot d},|d|=1$, and the unknown scattered field $u^{s}$ which is required to satisfy the Sommerfeld radiation condition [10]

$$
\lim _{r \rightarrow \infty} r\left(\frac{\partial u^{s}}{\partial r}-i k u^{s}\right)=0
$$

uniformly in $\hat{x}=x /|x|$ with $r=|x|$.

Furthermore it is known that the scattered field $u^{s}(x, d)$ has the following asymptotic representation

$$
u^{s}(x, d)=\gamma \frac{e^{i k|x|}}{|x|}\left\{u^{\infty}(\hat{x}, d)+O\left(\frac{1}{|x|}\right)\right\} \quad \text { as }|x| \rightarrow \infty
$$

uniformly for all directions $\hat{x}$, where $\gamma=\frac{1}{4 \pi}$ and the function $u^{\infty}(\hat{x}, d)$ defined on the unit sphere $S$ is known as the far field pattern with $\hat{x}$ and $d$ denoting, respectively, the observation direction and the incident direction.

The inverse problem we consider in this paper is, given the wave number $k$ and the far-field pattern $u^{\infty}(\hat{x}, d)$ for all $\hat{x}, d \in S$, to determine the location and shape of the crack and the refractive index $n$ or at least its compact support $D$.

The inverse scattering problem of identifying multiple obstacles has been extensively studied. We refer the reader to monograph [25] for a comprehen- 
sive study of the multiple scattering in general and the scattering by point-like scatterers in particular, where practical motivations of the corresponding models and historical facts are discussed. In 2007, the splitting method proposed in [16] is applicable to shape reconstruction for multiple obstacles. In 2008, some numerical methods for multiple acoustic scattering in the case of convex sound-soft obstacles were discussed by Antonie et al. [1] and references therein. In the same year, Ganesh and Hawkins in [11] generalized to the case of convex and non-convex multiple particles with different boundary condition and a few years later they developed a novel fast, high order, memory efficient algorithm to simulate multiple acoustic scattering induced by an ensemble with hundreds of particles in two space dimensions in [12]. In 2009, Cheng et al. [7] made using of the probe method to identify multiple obstacles and the type of boundary conditions for each obstacle. In 2012, Challa, et al. [5] have surveyed the direct and inverse electromagnetic scattering problem by a finite number of point-like obstacles. Recently, Kirsch and Liu [21] have studied the factorization method for identifying a mixed-type scatterer given as the union of a bounded impenetrable obstacle and a penetrable inhomogeneous medium with compact support. See [3] for the linear sampling method and $[13,14]$ for the factorization method of reconstruction for multiple scattering objects. For more scattering problems by multiple objects see $[6,17,18,19,20]$ and for the scattering by cracks with different faces we recommend the papers $[22,23]$.

In the present paper, we will apply the linear sampling method to recover the mixed-type scatterer which is composed by a crack and a penetrable inhomogeneous medium. To our knowledge, this is the first step in this direction although the multiple scattering problems have received a lot of attention as we mentioned above. The linear sampling method has been the subject of considerable attention since the method was formulated by Colton and Kirsch [9] for the first time, in the case of $2 \mathrm{D}$ obstacles with Dirichlet, Neumann and impedance boundary conditions and in the case of 2D inhomogeneous media. Until now, this method has been developed greatly and applied to solve a variety of inverse problems. For the mathematical judgement, the readers are advised to the article $[2,3]$. See [8] for a survey and overview. The main difficulty arising in reconstructing the mixed-type scatterer combined by a crack and a penetrable inhomogeneous medium by the linear sampling method is that the usual far field equation is not suitable for our problem. To this end, we need to analyze the properties of injectivity and denseness of some operators related to our complex scattering problem in some special Sobolev spaces and choose an appropriate far field equation. First of all, the unique and simultaneous determination result about the mixed-type scatterer must be established.

The remainder of the paper is organized as follows. In the next section, we present the forward problem and some key results on variational solutions to this problem. Section 3 is dedicated to the unique determination of the combined scatterer based on a mixed reciprocity relation. Then by introducing a novel far field equation, we investigate the recovery of the mixed-type scatterer by the linear sampling method in Section 4. 


\section{The Direct Scattering Problem}

This section is devoted to the solution of the direct acoustic scattering problem (1.1) and (1.2). In order to formulate our scattering problems more precisely we need to properly define the trace spaces on $\Gamma$. If $L^{2}(\partial \Omega), H^{1 / 2}(\partial \Omega)$ and $H^{-1 / 2}(\partial \Omega)$ denote the usual Sobolev spaces we define the following spaces:

$$
\begin{gathered}
H^{1 / 2}(\Gamma)=\left\{\left.u\right|_{\Gamma}: u \in H^{1 / 2}(\partial \Omega)\right\}, \\
\tilde{H}^{1 / 2}(\Gamma)=\left\{u \in H^{1 / 2}(\partial \Omega): \operatorname{supp} u \subseteq \bar{\Gamma}\right\}, \\
H^{-1 / 2}(\Gamma)=\left(\tilde{H}^{1 / 2}(\Gamma)\right)^{\prime}, \quad \text { the dual space of } \tilde{H}^{1 / 2}(\Gamma), \\
\tilde{H}^{-1 / 2}(\Gamma)=\left(H^{1 / 2}(\Gamma)\right)^{\prime}, \quad \text { the dual space of } H^{1 / 2}(\Gamma),
\end{gathered}
$$

and we have the chain

$$
\tilde{H}^{1 / 2}(\Gamma) \subset H^{1 / 2}(\Gamma) \subset L^{2}(\Gamma) \subset \tilde{H}^{-1 / 2}(\Gamma) \subset H^{-1 / 2}(\Gamma) .
$$

We make the following general assumptions throughout the paper.

Assumption. Assume that the boundary $\partial D$ of $D$ is smooth enough such that the imbedding of $H^{1}(D)$ into $L^{2}(D)$ is compact and the contrast function $q \in L^{\infty}(D)$ satisfies $\operatorname{Im} q \geq 0$ in $D$.

We extend $q$ by zero in $R^{3} \backslash \bar{\Gamma}$.

Sine the incident field $u^{i}$ satisfies the Helmholtz equation $\Delta u^{i}+k^{2} u^{i}=0$ in all of $R^{3}$, thus the scattered field $u^{s}$ solves the following problem with boundary data $f=-u^{i}$ and $g=-\frac{\partial u^{i}}{\partial \nu}-i \lambda u^{i}$ on $\Gamma$ and source term $h=-u^{i}$ in $D$. We consider a more general scattering problem for the scattered wave $u^{s}$ as following.

Remark. For a little confusion, we use $u$ to denote $u^{s}$.

The direct problem: Given $h \in L^{2}(D), f \in H^{\frac{1}{2}}(\Gamma)$ and $g \in H^{-\frac{1}{2}}(\Gamma)$ find $u \in H_{l o c}^{1}\left(R^{3} \backslash \bar{\Gamma}\right)$ such that

$$
\begin{cases}\Delta u+k^{2}(1+q) u=k^{2} q h & \text { in } R^{3} \backslash \bar{\Gamma}, \\ u_{+}=f \text { on }, \quad \frac{\partial u_{-}}{\partial \nu}+i \lambda u_{-}=g & \text { on } \Gamma, \\ \lim _{r \rightarrow \infty} r\left(\frac{\partial u}{\partial r}-i k u\right)=0, & r=|x| .\end{cases}
$$

The equation of (2.1) has to be understood in the variational sense, and the boundary conditions of (2.1) are understood in the sense of the trace operator. Here, and in the following, we do not distinguish between the function $q$ defined in $D$ and its extension by zero to all of $R^{3} \backslash \bar{\Gamma}$.

Theorem 1. For any $h \in L^{2}(D), f \in H^{\frac{1}{2}}(\Gamma)$ and $g \in H^{-\frac{1}{2}}(\Gamma)$, there exists at most one solution $u \in H_{\text {loc }}^{1}\left(R^{3} \backslash \overline{\bar{T}}\right)$ of $(2.1)$.

Proof. Clearly, it is sufficient to show that $u=0$ in $R^{3} \backslash \bar{\Gamma}$ if $h=0$ in $D$ and $f=g=0$ on $\Gamma$. Denote by $B_{R}$ a sufficiently large ball with radius $R$ containing 
$\bar{\Gamma}$ and $\bar{D}$ and by $S_{R}$ its boundary. From Green's theorem, the equation and boundary conditions of (2.1), we obtain

$$
\int_{S_{R}} u \frac{\partial \bar{u}}{\partial \nu} d s=\int_{B_{R} \backslash \bar{\Gamma}}\left[|\nabla u|^{2}-k^{2}(1+\bar{q})|u|^{2}\right] d x-i \int_{\Gamma} \lambda|u|^{2} d s .
$$

From this, since $k>0, \lambda<0$ and $\operatorname{Imq} \geq 0$, it follows that

$$
\operatorname{Im} \int_{S_{R}} u \frac{\partial \bar{u}}{\partial \nu} d s=k^{2} \int_{B_{R} \backslash \bar{\Gamma}} \operatorname{Im} q|u|^{2} d x-\int_{\Gamma} \lambda|u|^{2} d s \geq 0 .
$$

The Rellich's lemma [10] shows that $u=0$ in $R^{3} \backslash \bar{B}_{R}$ and it follows by the unique continuation principle [10] that $u=0$ in $R^{3} \backslash \bar{\Gamma}$.

Motivated by later use, we choose to adopt a variational approach in the study of the direct problem. For this purpose, we need to write it as an equivalent problem in a bounded domain and introduce $T_{R}: H^{\frac{1}{2}}\left(S_{R}\right) \rightarrow H^{-\frac{1}{2}}\left(S_{R}\right)$, the Dirichlet to Neumann operator (see [10]), defined by

$$
T_{R}(\varphi)=\frac{\partial \omega}{\partial \nu} \text { on } S_{R}
$$

with $\omega \in H_{l o c}^{1}\left(R^{3} \backslash \bar{B}_{R}\right)$ being the unique solution satisfying the Sommerfeld radiation condition and verifying

$$
\begin{cases}\Delta \omega+k^{2} \omega=0 & \text { in } R^{3} \backslash \bar{B}_{R} \\ \omega=\varphi & \text { on } S_{R}\end{cases}
$$

Let $\langle,\rangle_{S_{R}}$ denote the duality product between $H^{\frac{1}{2}}\left(S_{R}\right)$ and $H^{-\frac{1}{2}}\left(S_{R}\right)$ that coincides with $L^{2}\left(S_{R}\right)$ scalar product for regular functions. We have the following important properties of the Dirichlet to Neumann map [4].

Lemma 1. The Dirichlet to Neumann map $T_{R}$ is a bounded linear operator from $H^{\frac{1}{2}}\left(S_{R}\right)$ to $H^{-\frac{1}{2}}\left(S_{R}\right)$. Furthermore there exists a bounded operator $T_{0}: H^{\frac{1}{2}}\left(S_{R}\right) \rightarrow H^{-\frac{1}{2}}\left(S_{R}\right)$ satisfying

$$
-\left\langle T_{0} \varphi, \varphi\right\rangle_{S_{R}} \geq C\|\varphi\|_{H^{\frac{1}{2}}\left(S_{R}\right)}^{2}
$$

for some constant $C>0$, such that $T_{R}-T_{0}: H^{\frac{1}{2}}\left(S_{R}\right) \rightarrow H^{-\frac{1}{2}}\left(S_{R}\right)$ is compact.

The direct problem (2.1) can now be transformed into an equivalent problem, namely find $u \in H^{1}\left(B_{R} \backslash \bar{\Gamma}\right)$ such that

$$
\begin{cases}\Delta u+k^{2}(1+q) u=k^{2} q h & \text { in } B_{R} \backslash \bar{\Gamma}, \\ u_{+}=f, \quad \frac{\partial u_{-}}{\partial \nu}+i \lambda u_{-}=g & \text { on } \Gamma, \\ \frac{\partial u}{\partial \nu}=T_{R} u & \text { on } S_{R},\end{cases}
$$

where $h \in L^{2}(D), f \in H^{\frac{1}{2}}(\Gamma)$ and $g \in H^{-\frac{1}{2}}(\Gamma), T_{R}$ is the Dirichlet to Neumann map defined by (2.2). Referring to Theorem 2.1 in [4], we know that the problem (2.3) has at most one solution. We now show the following result on the well-posedness of the problem (2.3). 
Theorem 2. Assuming that the surface impedance $\lambda<0$, the boundary data $f \in H^{\frac{1}{2}}(\Gamma), g \in H^{-\frac{1}{2}}(\Gamma)$ and $h \in L^{2}(D)$, then the problem (2.3) has a unique solution $u \in H^{1}\left(B_{R} \backslash \bar{\Gamma}\right)$ such that

$$
\|u\|_{H^{1}\left(B_{R} \backslash \bar{\Gamma}\right)} \leq C\left(\|h\|_{L^{2}(D)}+\|f\|_{H^{\frac{1}{2}}(\Gamma)}+\|g\|_{H^{-\frac{1}{2}}(\Gamma)}\right)
$$

with a positive constant $C$ which is independent of $h, f$ and $g$ but on $\lambda, q$ and $B_{R}$.

Proof. Let $\tilde{f} \in H^{\frac{1}{2}}(\partial \Omega)$ be the extension of the Dirichlet date $f \in H^{\frac{1}{2}}(\Gamma)$ that satisfies $\|\tilde{f}\|_{H^{\frac{1}{2}}(\partial \Omega)} \leq C\|f\|_{H^{\frac{1}{2}}(\Gamma)}$ and let $u_{0} \in H^{1}\left(B_{R} \backslash \bar{\Omega}\right) \cap H^{1}(\Omega)$ be such that $\Delta u_{0}=0$ in $B_{R} \backslash \bar{\Omega}$ and $\Omega, u_{0}=0$ on $S_{R}$, and $u_{0}=\tilde{f}$ on $\partial \Omega$ and satisfy

$$
\left\|u_{0}\right\|_{H^{1}\left(B_{R} \backslash \bar{\Omega}\right) \cap H^{1}(\Omega)} \leq C\|f\|_{H^{\frac{1}{2}(\Gamma)}} .
$$

We now formulate (2.3) as a variational problem. To this end we define the Sobolev space

$$
X:=\left\{u \in H^{1}\left(B_{R} \backslash \bar{\Gamma}\right): u_{+}=0 \text { on } \Gamma\right\}
$$

Then $w:=u-u_{0} \in X$, where $u$ is a solution to (2.3). Furthermore $w$ satisfies

$$
\begin{cases}\Delta w+k^{2}(1+q) w=k^{2} q h-k^{2}(1+q) u_{0} & \text { in } B_{R} \backslash \bar{\Gamma} \\ w_{+}=0, \quad \frac{\partial w_{-}}{\partial \nu}+i \lambda w_{-}=g-\frac{\partial u_{0}}{\partial \nu}-i \lambda f & \text { on } \Gamma \\ \frac{\partial w}{\partial \nu}=T_{R} u-\frac{\partial u_{0}}{\partial \nu} & \text { on } S_{R} .\end{cases}
$$

The problem (2.3) can now be formulated in the following equivalent variational form: Find $u \in H^{1}\left(B_{R} \backslash \bar{\Gamma}\right)$ such that $w=u-u_{0} \in X$ and

$$
a(w, \phi)=L(\phi) \text { for all } \phi \in X,
$$

where the sesquilinear form $a(\cdot, \cdot): X \times X \rightarrow C$ is defined by

$$
a(w, \phi)=\int_{B_{R} \backslash \bar{\Gamma}}\left(\nabla w \cdot \nabla \bar{\phi}-k^{2}(1+q) w \bar{\phi}\right) d x-\int_{S_{R}} T_{R} w \bar{\phi} d s+i \lambda \int_{\Gamma} w_{-} \bar{\phi}_{-} d s,
$$

and the conjugate linear functional $L: X \rightarrow C$ is defined by

$$
\begin{aligned}
L(\phi)= & \int_{B_{R} \backslash \bar{\Gamma}} k^{2}\left[(1+q) u_{0}-q h\right] \bar{\phi} d x+\int_{S_{R}}\left(T_{R} u_{0}-\frac{\partial u_{0}}{\partial \nu}\right) \bar{\phi} d s \\
& +\int_{\Gamma} g[\bar{\phi}] d s-\int_{\Gamma} \frac{\partial u_{0-}}{\partial \nu} \bar{\phi}_{-} d s-i \lambda \int_{\Gamma} f \bar{\phi}_{-} d s,
\end{aligned}
$$

where the third integral of the above identity over $\Gamma$ is interpreted as the duality pairing between $H^{-\frac{1}{2}}(\Gamma)$ and $\tilde{H}^{\frac{1}{2}}(\Gamma)$ (note that $\phi_{+}=0$ on $\Gamma$ and $\left.[\bar{\phi}]:=\bar{\phi}_{-}-\bar{\phi}_{+} \in \tilde{H}^{\frac{1}{2}}(\Gamma)\right)$. 

where

Next we write $a(\cdot, \cdot)$ as the sum of two terms, i.e. $a(\cdot, \cdot)=a_{1}(\cdot, \cdot)+a_{2}(\cdot, \cdot)$,

$$
\begin{aligned}
& a_{1}(w, \phi)=\int_{B_{R} \backslash \bar{\Gamma}}(\nabla w \cdot \nabla \bar{\phi}+w \bar{\phi}) d x-\int_{S_{R}} T_{0} w \bar{\phi} d s+i \int_{\Gamma} \lambda w_{-} \bar{\phi}_{-} d s, \\
& a_{2}(w, \phi)=-\int_{B_{R} \backslash \bar{\Gamma}}\left[1+k^{2}(1+q)\right] w \bar{\phi} d x-\int_{S_{R}}\left(T_{R}-T_{0}\right) w \bar{\phi} d s .
\end{aligned}
$$

From the Cauchy-Schwarz inequality, the trace theorem, the properties of the Dirichlet to Neumann map $T_{R}$ and $T_{0}$ and the assumption on $p$, we have that

$$
\left|a_{1}(w, \phi)\right|,\left|a_{2}(w, \phi)\right| \leq C\left(\|w\|_{H^{1}\left(B_{R} \backslash \bar{\Gamma}\right)}\|\phi\|_{H^{1}\left(B_{R} \backslash \bar{\Gamma}\right)}\right) .
$$

Hence $a_{1}(\cdot, \cdot)$ and $a_{2}(\cdot, \cdot)$ are bounded sesquilinear forms.

Furthermore, noting that $\phi_{+}=0$ on $\Gamma$, the Green's first identity for $u_{0}$ and $\phi$ in the domain $B_{R} \backslash \bar{\Gamma}$ implies that

$$
-\int_{S_{R}} \frac{\partial u_{0}}{\partial \nu} \bar{\phi} d s-\int_{\Gamma} \frac{\partial u_{0-}}{\partial \nu} \bar{\phi}_{-} d s=-\int_{B_{R} \backslash \bar{\Gamma}} \nabla u_{0} \cdot \nabla \bar{\phi} d x .
$$

Therefore, again by the Cauchy-Schwarz inequality, the trace theorem and the properties of the Dirichlet to Neumann map $T_{R}$ and $T_{0}$ and the assumption on $p$, we have that

$$
\begin{aligned}
& |L(\phi)| \leq c_{1}\left\|u_{0}\right\|_{H^{1}\left(B_{R} \backslash \bar{\Gamma}\right)}\|\phi\|_{H^{1}\left(B_{R} \backslash \bar{\Gamma}\right)}+c_{2}\left\|u_{0}\right\|_{H^{\frac{1}{2}\left(S_{R}\right)}}\|\phi\|_{H^{\frac{1}{2}}\left(S_{R}\right)} \\
& \quad+c_{3}\|h\|_{L^{2}(D)}\|[\phi]\|_{L^{2}(D)}+c_{4}\|g\|_{H^{-\frac{1}{2}(\Gamma)}}\|[\phi]\|_{\tilde{H}^{\frac{1}{2}(\Gamma)}}+c_{5}\|f\|_{H^{\frac{1}{2}(\Gamma)}}\|\phi\|_{H^{\frac{1}{2}}(\Gamma)} \\
& \quad \leq C\left(\|h\|_{L^{2}(D)}+\|f\|_{H^{\frac{1}{2}(\Gamma)}}+\|g\|_{H^{-\frac{1}{2}(\Gamma)}}\right)\|\phi\|_{H^{1}\left(B_{R} \backslash \bar{\Gamma}\right)},
\end{aligned}
$$

which shows that $L$ is a bounded conjugate linear functional.

The coercivity of $a_{1}(\cdot, \cdot)$ is directly obtained form the property of $T_{0}$ and the assumption about $\lambda$. While, the compactness of $a_{2}(\cdot, \cdot)$ follows from the properties of $T_{R}-T_{0}$, the trace theorem and the compact imbedding theorem. By the Fredholm Theorem, the solvability of the variational problem (2.4) can be obtained by the injectivity of $a(\cdot, \cdot)$. The injectivity of $a(\cdot, \cdot)$ is equivalent to the fact that the only function $u \in X$ that satisfies $a(u, \phi)=0$ for all $\phi \in X$ is $u=0$. That is the uniqueness of a weak solution to the homogeneous boundary value problem of (2.1) which has be shown by Theorem 2.1. So we complete the proof of the theorem.

\section{The Unique Determination of the Mixed-Type Scat- terer}

This section is devoted to the uniqueness of the inverse problem, that is the unique determination of the location and shape of the union of the crack and the compact support of the inhomogeneous medium and the refractive index $n$.

Let us go back to the scattering problem (1.1) and (1.2). As incident fields $u^{i}$, plane waves and point sources are of special interest. Denote by 
$u^{s}(\cdot, d)$ the scattered field for an incident plane wave $u^{i}(\cdot, d)$ with the incident direction $d \in S$ and by $u^{\infty}(\cdot, d)$ the corresponding far-field pattern. The scattered field for an incident point source $\Phi(\cdot, z)$ which is the fundamental solution to the Helmholtz equation defined by

$$
\Phi(x, y)=\frac{e^{i k|x-y|}}{4 \pi(|x-y|)},
$$

with the source point $z \in R^{3}$ is denoted by $u^{s}(\cdot, z)$ and the corresponding far-field pattern by $\Phi^{\infty}(\cdot, z)$.

To establish the uniqueness result for the inverse scattering problem as mentioned above, we first give a generalization of the mixed reciprocity relation.

Lemma 2. For the scattering of plane wave $u^{i}(\cdot, d)$ with $d \in S$ and point source $\Phi(\cdot, z)$ with $z \in R^{3} \backslash(\bar{D} \cup \bar{\Gamma})$ by the mixed scatterer, we have

$$
\Phi^{\infty}(\hat{x}, z)=\gamma u^{s}(z,-\hat{x}), \quad z \in R^{3} \backslash(\bar{D} \cup \bar{\Gamma}), \hat{x} \in S,
$$

where $\gamma=\frac{1}{4 \pi}$.

Proof. By Green's second theorem and the Sommerfeld radiation condition we have that

$$
\begin{aligned}
0= & \int_{\partial D}\left(u^{s}(y, z) \frac{\partial u^{s}(y, d)}{\partial \nu(y)}-\frac{\partial u^{s}(y, z)}{\partial \nu(y)} u^{s}(y, d)\right) d s(y) \\
& +\int_{\Gamma}\left(u_{+}^{s}(y, z) \frac{\partial u_{+}^{s}(y, d)}{\partial \nu(y)}-\frac{\partial u_{+}^{s}(y, z)}{\partial \nu(y)} u_{+}^{s}(y, d)\right) d s(y) \\
& -\int_{\Gamma}\left(u_{-}^{s}(y, z) \frac{\partial u_{-}^{s}(y, d)}{\partial \nu(y)}-\frac{\partial u_{-}^{s}(y, z)}{\partial \nu(y)} u_{-}^{s}(y, d)\right) d s(y)
\end{aligned}
$$

for $z \in R^{3} \backslash(\bar{D} \cup \bar{\Gamma}), d \in S$. Using Green's representation formula [10], we obtain the representation

$$
\begin{aligned}
\Phi^{\infty}(\hat{x}, z)= & \gamma \int_{\partial D}\left(u^{s}(y, z) \frac{\partial e^{-i k \hat{x} \cdot y}}{\partial \nu(y)}-\frac{\partial u^{s}(y, z)}{\partial \nu(y)} e^{-i k \hat{x} \cdot y}\right) d s(y) \\
& +\gamma \int_{\Gamma}\left(u_{+}^{s}(y, z) \frac{\partial e^{-i k \hat{x} \cdot y}}{\partial \nu(y)}-\frac{\partial u_{+}^{s}(y, z)}{\partial \nu(y)} e^{-i k \hat{x} \cdot y}\right) d s(y) \\
& -\gamma \int_{\partial D}\left(u_{-}^{s}(y, z) \frac{\partial e^{-i k \hat{x} \cdot y}}{\partial \nu(y)}-\frac{\partial u_{-}^{s}(y, z)}{\partial \nu(y)} e^{-i k \hat{x} \cdot y}\right) d s(y)
\end{aligned}
$$

for $z \in R^{3} \backslash(\bar{D} \cup \bar{\Gamma}), d \in S$. Adding $\gamma$ times (3.1) with $d$ replaced by $-\hat{x}$ to equation (3.2) we have

$$
\begin{aligned}
\Phi^{\infty}(\hat{x}, z)= & \gamma \int_{\partial D}\left(u^{s}(y, z) \frac{\partial u(y,-\hat{x})}{\partial \nu(y)}-\frac{\partial u^{s}(y, z)}{\partial \nu(y)} u(y,-\hat{x})\right) d s(y) \\
& +\gamma \int_{\Gamma}\left(u_{+}^{s}(y, z) \frac{\partial u_{+}(y,-\hat{x})}{\partial \nu(y)}-\frac{\partial u_{+}^{s}(y, z)}{\partial \nu(y)} u_{+}(y,-\hat{x})\right) d s(y) \\
& -\gamma \int_{\Gamma}\left(u_{-}^{s}(y, z) \frac{\partial u_{-}(y,-\hat{x})}{\partial \nu(y)}-\frac{\partial u_{-}^{s}(y, z)}{\partial \nu(y)} u_{-}(y,-\hat{x})\right) d s(y)
\end{aligned}
$$


for $z \in R^{3} \backslash(\bar{D} \cup \bar{\Gamma}), d \in S$. Then by the boundary conditions of (1.1), we obtain

$$
\begin{aligned}
& \Phi^{\infty}(\hat{x}, z)=\gamma \int_{\partial D}\left(u^{s}(y, z) \frac{\partial u(y,-\hat{x})}{\partial \nu(y)}-\frac{\partial u^{s}(y, z)}{\partial \nu(y)} u(y,-\hat{x})\right) d s(y) \\
& +\gamma \int_{\Gamma} u_{+}^{s}(y, z) \frac{\partial u_{+}(y,-\hat{x})}{\partial \nu(y)} d s(y)+\gamma \int_{\Gamma}\left(i \lambda u_{-}^{s}(y, z)+\frac{\partial u_{-}^{s}(y, z)}{\partial \nu(y)}\right) u_{-}(y,-\hat{x}) d s(y) .
\end{aligned}
$$

On the other hand, Green's second theorem gives that for $z \in R^{3} \backslash(\bar{D} \cup \bar{\Gamma})$, $\hat{x} \in S$

$$
\gamma \int_{\partial D}\left(\frac{\partial \Phi(y, z)}{\partial \nu(y)} u^{i}(y,-\hat{x})-\Phi(y, z) \frac{\partial u^{i}(y,-\hat{x})}{\partial \nu(y)}\right) d s(y)=0 .
$$

Green's representation formula shows us that for $z \in R^{3} \backslash(\bar{D} \cup \bar{\Gamma}), \hat{x} \in S$

$$
\begin{aligned}
\gamma u^{s}(z,-\hat{x})= & \gamma \int_{\partial D}\left(\frac{\partial \Phi(y, z)}{\partial \nu(y)} u^{s}(y,-\hat{x})-\Phi(y, z) \frac{\partial u^{s}(y,-\hat{x})}{\partial \nu(y)}\right) d s(y) \\
& +\gamma \int_{\Gamma}\left(\frac{\partial \Phi(y, z)}{\partial \nu(y)} u_{+}^{s}(y,-\hat{x})-\Phi(y, z) \frac{\partial u_{+}^{s}(y,-\hat{x})}{\partial \nu(y)}\right) d s(y) \\
& -\gamma \int_{\Gamma}\left(\frac{\partial \Phi(y, z)}{\partial \nu(y)} u_{-}^{s}(y,-\hat{x})-\Phi(y, z) \frac{\partial u_{-}^{s}(y,-\hat{x})}{\partial \nu(y)}\right) d s(y) .
\end{aligned}
$$

The sum of the equation (3.4) and (3.5) is

$$
\begin{aligned}
\gamma u^{s}(z,-\hat{x})= & \gamma \int_{\partial D}\left(\frac{\partial \Phi(y, z)}{\partial \nu(y)} u(y,-\hat{x})-\Phi(y, z) \frac{\partial u(y,-\hat{x})}{\partial \nu(y)}\right) d s(y) \\
& +\gamma \int_{\Gamma}\left(\frac{\partial \Phi(y, z)}{\partial \nu(y)} u_{+}(y,-\hat{x})-\Phi(y, z) \frac{\partial u_{+}(y,-\hat{x})}{\partial \nu(y)}\right) d s(y) \\
& -\gamma \int_{\Gamma}\left(\frac{\partial \Phi(y, z)}{\partial \nu(y)} u_{-}(y,-\hat{x})-\Phi(y, z) \frac{\partial u_{-}(y,-\hat{x})}{\partial \nu(y)}\right) d s(y) .
\end{aligned}
$$

Then the boundary conditions of (1.1) imply that

$$
\begin{aligned}
\gamma u^{s}(z,-\hat{x})= & \gamma \int_{\partial D}\left(\frac{\partial \Phi(y, z)}{\partial \nu(y)} u(y,-\hat{x})-\Phi(y, z) \frac{\partial u(y,-\hat{x})}{\partial \nu(y)}\right) d s(y) \\
& -\gamma \int_{\Gamma} \Phi(y, z) \frac{\partial u_{+}(y,-\hat{x})}{\partial \nu(y)} d s(y) \\
& -\gamma \int_{\Gamma}\left(\frac{\partial \Phi(y, z)}{\partial \nu(y)}+i \lambda \Phi(y, z)\right) u_{-}(y,-\hat{x}) d s(y) .
\end{aligned}
$$

Thus, from (3.3) and (3.6) our assertion can be deduced from the boundary conditions for the scattered field $u^{s}(x, z)$ due to the incident point source $\Phi(x, z)$.

Using the generalized mixed reciprocity relation, the following uniqueness result about the shape and location of the crack and the inhomogeneous medium can be proved. 
Theorem 3. Assume that $u_{1}^{s}(x, z)$ and $u_{2}^{s}(x, z)$ are the two scattered fields corresponding to incident point source $\Phi(x, z)$ for the two mixed-type scatterers constituted by a crack $\Gamma_{1}$, an inhomogeneous medium with compact support $D_{1}$ and a crack $\Gamma_{2}$, an inhomogeneous medium with compact support $D_{2}$, respectively. If the far field pattern of the scattered field of (1.1) and (1.2) for the same incident plane wave $u^{i}=e^{i k x \cdot d}$ coincide at a fixed wave number for all incident directions $d \in S$ and observation directions $\hat{x} \in S$, then $\Gamma_{1}=\Gamma_{2}$ and $D_{1}=D_{2}$.

Proof. If the two mixed-type scatterers are different, there are three possibilities: (1) $D_{1}=D_{2}=D, \Gamma_{1} \neq \Gamma_{2}$; (2) $D_{1} \neq D_{2}, \Gamma_{1}=\Gamma_{2}=\Gamma$; (3) $D_{1} \neq D_{2}$, $\Gamma_{1} \neq \Gamma_{2}$. We give the proofs for the case (1) and (2), the result for situation (3) can be obtained similarly.

Case (1) $D_{1}=D_{2}=D, \Gamma_{1} \neq \Gamma_{2}$. Let $G$ be the unbounded component of $R^{3} \backslash\left(\bar{D} \cup \bar{\Gamma}_{1} \cup \bar{\Gamma}_{2}\right)$. By Rellich's lemma the scattered field $u^{s}(\cdot,-\hat{x})$ corresponding to the incident plane wave $u^{i}(\cdot,-\hat{x})$ coincide in the unbounded domain $G$.

Without loss of generality, there exists $z^{*} \in \Gamma_{1}$ but $z^{*} \notin \Gamma_{2}$. Choose $h>0$ such that the sequence $z_{j}=z^{*}+\frac{h}{j} \nu\left(z^{*}\right), j=1,2, \cdots$ is contained in $G$, where the unit normal vector to the crack $\Gamma_{1}$ at $z^{*}$. Consider the solution $u^{s}\left(\cdot, z_{j}\right)$ to the problem (1.1) due to the incident point source $\Phi\left(\cdot, z_{j}\right)$. By lemma 3.1, the far field $\Phi^{\infty}\left(\cdot, z_{j}\right)$ coincide. Then the Rellich's lemma implies that $u_{1}^{s}\left(x, z_{j}\right)=u_{2}^{s}\left(x, z_{j}\right)$ for $x \in G$.

Consider $u_{j}^{s}\left(x, z_{j}\right)=u_{2}^{s}\left(x, z_{j}\right)$ as the scattered field corresponding to $D$ and $\Gamma_{2}$. Note that $u_{j+}^{s}(\cdot)=-\Phi\left(\cdot, z_{j}\right)$ and $\frac{\partial u_{j-}^{s}(\cdot)}{\partial \nu}+i \lambda u_{j-}^{s}(\cdot)=-\frac{\partial \Phi\left(\cdot, z_{j}\right)}{\partial \nu}+$ $i \lambda \Phi\left(\cdot, z_{j}\right)$ on $\Gamma_{2}$ are uniformly bounded. By the well-posedness of the direct problem we have that $\left\|u_{j}^{s}\left(z^{*}\right)\right\|_{H_{l o c}^{1}\left(R^{3} \backslash\left(\bar{D} \cup \bar{\Gamma}_{2}\right)\right)}$ is bounded as $j \rightarrow \infty$, whence from the trace theorem $\left\|u_{j}^{s}\left(z^{*}\right)\right\|_{H^{\frac{1}{2}}\left(B_{r}\left(z^{*}\right) \cap \Gamma_{1}\right)}$ is uniformly bounded respect to $j$, where $B_{r}\left(z^{*}\right)$ is a small neighborhood centered at $z^{*}$ not intersecting $\Gamma_{2}$ and $D$.

On the other hand, considering $u_{j}^{s}\left(x, z_{j}\right)=u_{1}^{s}\left(x, z_{j}\right)$ as the solution corresponding to $D$ and $\Gamma_{1}$, from the boundary condition $u_{j+}^{s}(\cdot)=-\Phi\left(\cdot, z_{j}\right)$ on the crack $\Gamma_{1}$, we have that $\left\|u_{j}^{s}\right\|_{H^{\frac{1}{2}}\left(B_{r}\left(z^{*}\right) \cap \Gamma_{1}\right)} \rightarrow \infty$ as $j \rightarrow \infty$ since $\left\|\Phi\left(\cdot, z_{j}\right)\right\|_{H^{\frac{1}{2}}\left(B_{r}\left(z^{*}\right) \cap \Gamma_{1}\right)} \rightarrow \infty$ as $j \rightarrow \infty$. This is a contradiction. Therefore $\Gamma_{1}=\Gamma_{2}$.

Case (2) $D_{1} \neq D_{2}, \Gamma_{1}=\Gamma_{2}=\Gamma$. We refer the reader to [27] where the unique determination of the penetrable, inhomogeneous obstacle from the farfield pattern for all incident plane waves at a fixed frequency. By an analogous argument and technique, one can prove that $D_{1}=D_{2}$.

Remark. For the inverse penetrable obstacle scattering problem, the transmission boundary condition is usually defined as $u_{+}=u_{-}, \frac{\partial u_{+}}{\partial \nu}=\mu \frac{\partial u_{-}}{\partial \nu}$ on the transmission boundary. It well-known that there are some uniqueness results for the penetrable obstacle under the assumption that $\mu \neq 1$. In the paper [27], based on constructing a well-posed interior transmission problem in a small domain associated with the Helmholtz equation and a priori estimates of solution to the transmission scattering problem with boundary data, a new method is 
proposed to prove the unique determination of the penetrable, inhomogeneous obstacle with $\mu=1$. This method can be applied directly to our inverse problem to uniquely determine the domain $D$ for the case (2) without any difficulty.

Based on [24], we show the uniqueness of the refractive index $n$ by using the following two lemmas.

Lemma 3. Let $u(\cdot, d)$ be the solution to the problem (1.1) and (1.2) due to the incident plane waves $u^{i}(\cdot, d)$ with incident direction $d \in S$, and assume that $k^{2}$ is not the Neumann eigenvalue of the equation $\Delta v+k^{2} n v=0$ in $D$, then the set $\left\{\left(\left.\frac{\partial u(\cdot, d)}{\partial \nu}\right|_{\partial D},\left.u_{-}(\cdot, d)\right|_{\Gamma}\right): d \in S\right\}$ has dense range in $W:=H^{-\frac{1}{2}}(\partial D) \times H^{\frac{1}{2}}(\Gamma)$.

Proof. Notice that $B_{R}$ is a sufficiently large ball containing $\bar{\Gamma}$ and $\bar{D}$ with radius $R$ and boundary $S_{R}$. We may assume that $k^{2}$ is not a Dirichlet eigenvalue for the bounded domain $B_{R}$. Then the set $\left\{u^{i}(\cdot, d): d \in S\right\}$ is complete in $H^{\frac{1}{2}}\left(S_{R}\right)$ (see [4]). Thus we just need to prove that the operator $\Lambda: H^{\frac{1}{2}}\left(S_{R}\right) \rightarrow$ $W$ defined by

$$
\Lambda \phi=\left(\left.\frac{\partial u}{\partial \nu}\right|_{\partial D},\left.u_{-}\right|_{\Gamma}\right)
$$

has dense range. Here $u$ is the solution of the problem (1.1) and (1.2), and $\phi$ is the Dirichlet boundary data of the problem

$$
\begin{cases}\Delta u^{i}+k^{2} u^{i}=0 & \text { in } B_{R} \\ u^{i}=\phi & \text { on } S_{R} .\end{cases}
$$

Making use of the Green's second theorem, we obtain that the adjoint operator of $\Lambda$ is $\Lambda^{*}: W^{*} \rightarrow H^{-\frac{1}{2}}\left(S_{R}\right)$ defined by

$$
\Lambda^{*}(\varphi, \alpha)=\left.\overline{\left(\frac{\partial v}{\partial \nu}-\frac{\partial \tilde{v}}{\partial \nu}\right)}\right|_{S_{R}}, \quad(\varphi, \alpha) \in W^{*},
$$

where $W^{*}:=H^{\frac{1}{2}}(\partial D) \times \tilde{H}^{-\frac{1}{2}}(\Gamma), v$ satisfies the problem

$$
\begin{cases}\Delta v+k^{2} n v=0 & \text { in } D, \\ \Delta v+k^{2} v=0 & \text { in } R^{3} \backslash(\bar{D} \cup \bar{\Gamma}), \\ v_{+}-v_{-}=\bar{\varphi}, \quad \frac{\partial v_{+}}{\partial \nu}-\frac{\partial v_{-}}{\partial \nu}=0 & \text { on } \partial D, \\ v_{+}=0, \quad \frac{\partial v_{+}}{\partial \nu}-\frac{\partial v_{-}}{\partial \nu}-i \lambda v_{-}=\bar{\alpha}, & \text { on } \Gamma, \\ \lim _{r \rightarrow \infty} r\left(\frac{\partial v}{\partial r}-i k v\right)=0, & r=|x|\end{cases}
$$

and $\tilde{v}$ is a solution of the following problem

$$
\begin{cases}\Delta \tilde{v}+k^{2} \tilde{v}=0 & \text { in } B_{R} \backslash \bar{\Gamma}, \\ \tilde{v}_{+}=0, \quad \frac{\partial \tilde{v}_{+}}{\partial \nu}-\frac{\partial \tilde{v}_{-}}{\partial \nu}-i \lambda \tilde{v}_{-}=0 & \text { on } \Gamma, \\ \tilde{v}=v & \text { on } S_{R} .\end{cases}
$$

Remark. The well-posedness of the problem (3.7) can be obtained by an analogous derivation as the problem (1.1) and (1.2) and a similar problem as (3.8) has been proved in [15]. 
Now we only need to prove that the operator $\Lambda^{*}$ is injective. Letting $\Lambda^{*}(\varphi, \alpha)=0$, then we have

$$
\frac{\partial v}{\partial \nu}=\frac{\partial \tilde{v}}{\partial \nu}, \quad v=\tilde{v} \quad \text { on } S_{R}
$$

We define

$$
w= \begin{cases}\tilde{v} & \text { in } B_{R} \\ v & \text { in } R^{3} \backslash \bar{B}_{R}\end{cases}
$$

Then $w$ satisfies the Helmholtz equation $\Delta w+k^{2} w=0$ in $R^{3}$ and the Sommerfeld radiation condition, hence $w=0$ in $R^{3}$. The unique continuation principle shows us that $v=0$ in $B_{R} \backslash(\bar{D} \cup \bar{\Gamma})$, thus the boundary conditions of problem (3.7) imply that $\alpha=0$ and $\frac{\partial v_{-}}{\partial \nu}=\frac{\partial v_{+}}{\partial \nu}=0$. Because $k^{2}$ is not the Neumann eigenvalue of the equation $\Delta v+k^{2} n v=0$ in $D$, so $v=0$ for $x \in D$. Thus again by the boundary condition in (3.7) we have $\varphi=0$. Therefore we have shown that $\Lambda^{*}$ is injective. The lemma has been proved.

Based on the above lemma we can deduce the following orthogonal relation.

Lemma 4. Assume that the location and shape of the mixed-type scatterer are known and $k^{2}$ is not the Neumann eigenvalue of the equation $\Delta u+k^{2} n u=0$ in D. For the scattering of different refractive indices $n_{1}$ and $n_{2}$ due to the incident plane waves $u^{i}(\cdot, d)$ with incident direction $d \in S$, let $u_{1}(\cdot, d)$ and $u_{2}(\cdot, d)$ be the solution to problem (1.1) and (1.2) with respect to $n_{1}$ and $n_{2}$, suppose that the corresponding far field pattern $u_{1}^{\infty}(\hat{x}, d)=u_{2}^{\infty}(\hat{x}, d)$ for $\hat{x}, d \in S$. Let $u_{1}, u_{2} \in H^{1}(D)$ satisfy the following equations

$$
\Delta u_{1}+k^{2} n_{1} u_{1}=0, \quad \Delta u_{2}+k^{2} n_{2} u_{2}=0 \quad \text { in } D,
$$

then we have the orthogonal relation:

$$
\int_{D}\left(n_{1}-n_{2}\right) u_{1} u_{2} d x=0 .
$$

Proof. First we show that (3.9) holds true for $u_{1}(\cdot, d)$ and $u_{2}(x)$. By Rellich's lemma we have from $u_{1}^{\infty}(\hat{x}, d)=u_{2}^{\infty}(\hat{x}, d)$ for $\hat{x}, d \in S$ that

$$
u_{1}(\cdot, d)=u_{2}(\cdot, d), \quad \frac{\partial u_{1}(\cdot, d)}{\partial \nu}=\frac{\partial u_{2}(\cdot, d)}{\partial \nu} \quad \text { on } \partial D
$$

Whereby, and by the fact that $u_{1}(\cdot, d), u_{2}(\cdot, d)$ and $u_{2}$ satisfy the equation $\Delta v+k^{2} n v=0$ in $D$, we have

$$
\begin{gathered}
\int_{D}\left(n_{1}-n_{2}\right) u_{1}(x, d) u_{2}(x) d x=-\frac{1}{k^{2}} \int_{D}\left(\Delta u_{1}(x, d)+k^{2} n_{2} u_{1}(x, d)\right) u_{2}(x) d x \\
=-\frac{1}{k^{2}} \int_{D}\left[\Delta u_{1}(x, d)-\Delta u_{2}(x, d)+k^{2} n_{2}\left(u_{1}(x, d)-u_{2}(x, d)\right)\right] u_{2}(x) d x \\
=-\frac{1}{k^{2}} \int_{D}\left(u_{1}(x, d)-u_{2}(x, d)\right)\left(\Delta u_{2}+k^{2} n_{2} u_{2}\right) d x=0,
\end{gathered}
$$


where Green's second theorem has been used for the third equality.

Next our assertion will be obtained if we could show that the solution $u$ can be approximated by $u(\cdot, d)$ in $L^{2}(D)$. In contrast, if $\{u(\cdot, d): d \in S\}$ has no dense range in $U:=\left\{u \in H^{1}(D): \Delta u+k^{2} n u=0\right\}$, then by Hahn-Banach theorem, there exists a function $f \in L^{2}(D)$ such that

$$
\int_{D} f(x) u(x, d) d x=0, \quad d \in S,
$$

and also has a function $u \in U$ such that

$$
\int_{D} f(x) u(x) d x \neq 0
$$

Let $v \in H^{1}(D)$ be a solution to the following problem

$$
\Delta v+k^{2} n v=f \quad \text { in } D, \quad \frac{\partial v}{\partial \nu}=0 \quad \text { on } \partial D .
$$

From (3.10) and (3.12), we have

$$
0=\int_{D} f(x) u(x, d) d x=\int_{D}\left(\Delta v+k^{2} n v\right) u(x, d) d x=\int_{\partial D} v \frac{\partial u(x, d)}{\partial \nu} d s
$$

by Green's second theorem. Lemma 3.2 shows us that $v(x)=0$, for $x \in \partial D$. Thus we have

$$
\int_{D} f(x) u(x) d x=\int_{D}\left(\Delta v+k^{2} n v\right) u(x) d x=\int_{D} v(x)\left(\Delta u+k^{2} n u\right) d x=0 .
$$

This is a contradiction to equality (3.11). Thus the lemma is proved.

Now the uniqueness result about the refractive index $n$ is presented in the following.

Theorem 4. Assume that $\Gamma$ and $\partial D$ are given, $k^{2}$ is not the Neumann eigenvalue of the equation $\Delta u+k^{2} n u=0$ in $D$. Then the refractive index $n$ can be uniquely determined by the far field pattern $u^{\infty}(\hat{x}, d)$, for $\hat{x}, d \in S$.

Proof. Uhlmann [26] has proved that $u_{1} u_{2}$ is complete in $L^{2}(D)$. So the theorem can be obtained by Lemma 3.3.

\section{The Linear Sampling Method}

In this part, we will adapt the linear sampling method developed for scattering from objects with nonempty interior to solve the inverse problem, that is to determine the location and shape of the crack and the compact support $D$ of the penetrable inhomogeneous medium and give a mathematical justification of the method. We start with some preparations and introduction. 
We rewrite the problem $(2.1)$ as

$$
\begin{cases}\Delta u+k^{2}(1+q) u=k^{2} \frac{q}{\sqrt{|q|}} \tilde{h} & \text { in } R^{3} \backslash \bar{\Gamma}, \\ u_{+}=f, \quad \frac{\partial u_{-}}{\partial \nu}+i \lambda u_{-}=g & \text { on } \Gamma, \\ \lim _{r \rightarrow \infty} r\left(\frac{\partial u}{\partial r}-i k u\right)=0, & r=|x|\end{cases}
$$

with $\tilde{h} \in X_{q}(D)$ which will be defined in the following paragraph, $f \in H^{\frac{1}{2}}(\Gamma)$ and $g \in H^{-\frac{1}{2}}(\Gamma)$. Notice that the problem (1.1) and (1.2) is the special case of (4.1) with boundary data $f=-u^{i}$ and $g=-\frac{\partial u^{i}}{\partial \nu}-i \lambda u^{i}$ on $\Gamma$ and source $\operatorname{term} \tilde{h}=-\sqrt{|q|} u^{i}$ in $D$.

A Herglotz wave function is a solution of the Helmholtz equation in $R^{3}$ of the form

$$
v_{\sigma}=\int_{S} e^{i k x \cdot d} \sigma(d) d s(d)
$$

with density $\sigma \in L^{2}(S)$. As an auxiliary operator, we define $\mathcal{H}: L^{2}(S) \rightarrow$ $H^{*}:=X_{q}(D) \times H^{1 / 2}(\Gamma) \times H^{-1 / 2}(\Gamma)$ by

$$
\mathcal{H} \pi=\left(\left.\sqrt{|q|} v_{\sigma}\right|_{D},\left.v_{\sigma}^{+}\right|_{\Gamma},\left.\left(\frac{\partial v_{\sigma}}{\partial \nu}+i \lambda v_{\sigma}^{-}\right)\right|_{\Gamma}\right)
$$

where $v_{\sigma}^{ \pm}(x)=\lim _{h \rightarrow 0} v_{\sigma}(x \pm h \nu)$ for $x \in \Gamma, \frac{\partial v_{\sigma}^{ \pm}}{\partial \nu}=\lim _{h \rightarrow 0} \nu \cdot \nabla v_{\sigma}(x \pm h \nu)$ for $x \in \Gamma$, and $X_{q}(D):=\sqrt{|q|} \bar{X}, \bar{X}$ is the closure of the set of the Herglotz wave functions in $L^{2}(D)$.

We define the data-to-pattern operator $B: H^{*} \rightarrow L^{2}(S)$ by

$$
B(\tilde{h}, f, g)(\hat{x})=u^{\infty}
$$

where $u^{\infty}$ is the far field pattern of the scattered field $u$ of problem (4.1) with data $(\tilde{h}, f, g) \in H^{*}$. The far field operator $F: L^{2}(S) \rightarrow L^{2}(S)$ is defined by

$$
(F \sigma)(\hat{x})=\int_{S} u^{\infty}(\hat{x}, d) \sigma(d) d s(d)
$$

where $u^{\infty}$ is the far field pattern of the scattered wave $u^{s}$ of problem (1.1) and (1.2) for incident plane wave $u^{i}$. By superposition we have the following relation: $(F \sigma)=-B(\mathcal{H} \sigma)$.

Remark. For the inverse scattering problems by obstacles, the right hand side of the far field equation is usually chosen as a point source or its far field pattern. For some special scatterer, taking the crack as an example, one usually chooses an integral on some open arc as the right hand side of the far field equation. We are inspired by the latter and will prove that it is valid for the linear sampling method.

The linear sampling method looks for a solution $\sigma \in L^{2}(S)$ of the far field equation

$$
(F)(\sigma)(\hat{x})=Q^{\infty}(\hat{x}) \quad \text { for } \sigma \in L^{2}(S), \hat{x} \in S,
$$


where $Q^{\infty}(\hat{x}) \in L^{2}(S)$ is the far field pattern of the potential $Q(x)$ defined by

$$
\begin{aligned}
Q(x)= & \int_{\Sigma}|q(y)| \delta_{\Sigma}(y) \Phi(x, y) d s(y)+\int_{L} \alpha_{L}(y) \Phi(x, y) d s(y) \\
& +\int_{L} \beta_{L}(y) \frac{\partial \Phi(x, y)}{\partial \nu(y)} d s(y)
\end{aligned}
$$

with $\delta_{\Sigma} \in \bar{X}(\Sigma)$ for any open bounded domain $\Sigma$ with smooth boundary, and $\alpha_{L} \in \tilde{H}^{-1 / 2}(L)$ and $\beta_{L} \in \tilde{H}^{1 / 2}(L)$ for any smooth non intersecting open arc $L$ such that $L \cap \Sigma=\emptyset$. We will characterize the mixed-type scatterer by the behavior of an approximate solution $\sigma$ of the far field equation (4.3).

We now define the compact operator $\mathcal{F}: H:=X_{q}(D) \times \tilde{H}^{-1 / 2}(\Gamma) \times$ $\tilde{H}^{1 / 2}(\Gamma) \rightarrow L^{2}(S)$ by

$$
\begin{aligned}
\mathcal{F}(\delta, \alpha, \beta)(\hat{x})= & \gamma \int_{D}|q(y)| \delta(y) e^{-i k \hat{x} \cdot y} d s(y)+\gamma \int_{\Gamma} \alpha(y) e^{-i k \hat{x} \cdot y} d s(y) \\
& +\gamma \int_{\Gamma} \beta(y) \frac{\partial e^{-i k \hat{x} \cdot y}}{\partial \nu(y)} d s(y), \quad \hat{x} \in S
\end{aligned}
$$

with densities $\delta \in \bar{X}(D), \alpha \in \tilde{H}^{-1 / 2}(\Gamma)$ and $\beta \in \tilde{H}^{1 / 2}(\Gamma)$ and the constant $\gamma=\frac{1}{4 \pi}$. The function $\mathcal{F}(\delta, \alpha, \beta)(\hat{x})$ is the far field pattern of the radiating solution $P(\delta, \alpha, \beta)(x)$ of the Helmholtz equation in $R^{3} \backslash \bar{\Gamma}$ where $P(\delta, \alpha, \beta)$ is defined by

$$
\begin{aligned}
P(\delta, \alpha, \beta)(x)= & \int_{D}|q(y)| \delta(y) \Phi(x, y) d s(y)+\int_{\Gamma} \alpha(y) \Phi(x, y) d s(y) \\
& +\int_{\Gamma} \beta(y) \frac{\partial \Phi(x, y)}{\partial \nu(y)} d s(y) .
\end{aligned}
$$

For latter use, we define the operators $S, K, K^{\prime}, T$ and $V$ as

$$
\begin{aligned}
& S \varphi(x)=\int_{\Gamma} \varphi(y) \Phi(x, y) d s(y), \quad K \varphi(x)=\int_{\Gamma} \varphi(y) \frac{\Phi(x, y)}{\partial \nu(y)} d s(y), \quad x \in \Gamma, \\
& K^{\prime} \varphi(x)=\int_{\Gamma} \varphi(y) \frac{\partial \Phi(x, y)}{\partial \nu(x)} d s(y), V \phi(x)=\int_{D}|q(y)| \phi(y) \Phi(x, y) d s(y), x \in \Gamma, \\
& T \varphi(x)=\frac{\partial}{\partial \nu(x)} \int_{\Gamma} \varphi(y) \frac{\partial \Phi(x, y)}{\partial \nu(y)} d s(y), \quad x \in \Gamma .
\end{aligned}
$$

For the mapping properties of these potentials see [4].

It is easy to check that $P$ is a radiation solution of the following problem:

$$
\begin{aligned}
& \Delta P+k^{2}(1+q) P=k^{2} \frac{q}{\sqrt{|q|}}\left(\sqrt{|q|} P-\frac{|q|^{3 / 2}}{k^{2} q} \delta\right) \quad \text { in } R^{3} \backslash \bar{\Gamma} \\
& P_{+}=V \delta+S \alpha+K \beta+\frac{1}{2} \beta \quad \text { on } \Gamma \\
& \frac{\partial P_{-}}{\partial \nu}+i \lambda P_{-}=\frac{\partial}{\partial \nu}(V \delta)+K^{\prime} \alpha+\frac{1}{2} \alpha+T \beta \\
& \quad+i \lambda\left(V \delta+S \alpha+K \beta-\frac{1}{2} \beta\right) \quad \text { on } \Gamma .
\end{aligned}
$$


We set

$$
\left(\begin{array}{l}
\tilde{h}_{1} \\
f_{1} \\
g_{1}
\end{array}\right)=M\left(\begin{array}{l}
\delta \\
\alpha \\
\beta
\end{array}\right)=E M^{\prime}\left(\begin{array}{l}
\delta \\
\alpha \\
\beta
\end{array}\right)+\left(\begin{array}{c}
\sqrt{|q|} P \\
0 \\
0
\end{array}\right)
$$

where

$$
E=\left(\begin{array}{ccc}
I & 0 & 0 \\
0 & I & 0 \\
0 & i \lambda I & -I
\end{array}\right) \quad M^{\prime}=\left(\begin{array}{ccc}
-\frac{|q|^{3 / 2}}{k^{2} q} & 0 & 0 \\
V & S & K+\frac{1}{2} I \\
-\frac{\partial}{\partial \nu} V & -K^{\prime}-\frac{1}{2} I & -T+i \lambda I
\end{array}\right)
$$

Then, $\mathcal{F}(\hat{x})=B\left(\tilde{h}_{1}, f_{1}, g_{1}\right)(\hat{x})=B M(\delta, \alpha, \beta)$. By using the analogous idea in Chapter 8 [4], we can show that $M: H \rightarrow H^{*}$ is bounded with a bounded inverse $M^{-1}$. So the far field operator can be factorized as

$$
(F \sigma)=-\mathcal{F} M^{-1} \mathcal{H} \sigma, \quad \sigma \in L^{2}(S) .
$$

To prove the existence of an approximate solution of (4.3), we first explore the properties of the operator $\mathcal{H}$ and $\mathcal{F}$. To this end we have to exclude the case that $k^{2}$ is the eigenvalue of an eigenvalue problem.

DEFINITION $1 . k^{2}$ is called an interior transmission eigenvalue if there exists $(u, w) \in H_{0}^{1}(D) \times L^{2}(D,|q| d x)$ with $(u, w) \neq(0,0)$ and a sequence $\left\{w_{j}\right\}$ in $H^{2}(D)$ with $w_{j} \rightarrow w$ in $L^{2}(D,|q| d x)$ and $\Delta w_{j}+k^{2} w_{j}=0$ in $D$ and

$$
\int_{D}\left[\nabla u \cdot \nabla \psi-k^{2} u \psi\right] d x=\int_{D}|q| w \psi d x \quad \text { for all } \psi \in H^{1}(D) .
$$

The weighted space $L^{2}(D,|q| d x)$ is defined as the completion of $L^{2}(D)$ with respect to the norm corresponding to the inner product $(\phi, \varphi)_{L^{2}(D,|q| d x)}=$ $\int_{D}|q| \phi \bar{\varphi} d x$.

Lemma 5. Assume that $k^{2}$ is not the interior transmission eigenvalue in $D$, then the range of the operator $\mathcal{H}: L^{2}(S) \rightarrow H^{*}$ is dense.

Proof. It is sufficient to show that the adjoint operator $\mathcal{H}^{*}: H \rightarrow L^{2}(S)$ is injective.

In order to characterize the adjoint operator we recall that $\mathcal{H}^{*}$ is defined by

$$
\langle\mathcal{H} \sigma,(\sqrt{|q|} \delta, \alpha, \beta)\rangle=\left\langle\sigma, \mathcal{H}^{*}(\sqrt{|q|} \delta, \alpha, \beta)\right\rangle
$$

for $\sigma \in L^{2}(S)$ and $(\sqrt{|q|} \delta, \alpha, \beta) \in H$ with $\delta \in \bar{X}(D), \alpha \in \tilde{H}^{-1 / 2}(\Gamma)$ and $\beta \in \tilde{H}^{1 / 2}(\Gamma)$. Note that the left hand side is the duality pairing between $H^{*}$ and $H$ while the right hand side is the $L^{2}(S)$-inner product. One can easily see from the above identity by changing the order of integration that $\mathcal{H}^{*}(\sqrt{|q|} \delta, \alpha, \beta)$ is given as

$$
\begin{aligned}
\mathcal{H}^{*}(\sqrt{|q|} \delta, \alpha, \beta)= & \int_{D}|q(x)| \delta(x) e^{-i k x \cdot d} d s(x)+\int_{\Gamma} \alpha(x) e^{-i k x \cdot d} d s(x) \\
& +\int_{\Gamma} \beta(x) \frac{\partial e^{-i k x \cdot d}}{\partial \nu(x)} d s(x)+i \lambda \int_{\Gamma} \beta(x) e^{-i k x \cdot d} d s(x)
\end{aligned}
$$


with $d \in S$. Hence $\mathcal{H}^{*}(\sqrt{|q|} \delta, \alpha, \beta)$ coincides with the far field pattern of the potential

$$
\begin{aligned}
\gamma^{-1} W(z)= & \int_{D}|q(x)| \delta(x) \Phi(z, x) d s(x)+\int_{\Gamma} \alpha(x) \Phi(z, x) d s(x) \\
& +\int_{\Gamma} \beta(x) \frac{\partial \Phi(z, x)}{\partial \nu(x)} d s(x)+i \lambda \int_{\Gamma} \beta(x) \Phi(z, x) d s(x), \quad z \in R^{3} \backslash \bar{\Gamma} .
\end{aligned}
$$

This potential $W \in H_{l o c}^{1}\left(R^{3} \backslash \bar{\Gamma}\right)$ satisfies the Helmholtz equation (4.5) in $R^{3} \backslash \bar{\Gamma}$ and the Sommerfeld radiation condition.

Now assume that $\mathcal{H}^{*}(\sqrt{|q|} \delta, \alpha, \beta)=0$. This means that the far field pattern of $W$ is zero, then from Rellich's lemma we conclude that $W=0$ in $R^{3} \backslash(\bar{D} \cup \bar{\Gamma})$. Using the jump relations of single-and double-layer potentials across $\Gamma$, we obtain that

$$
\gamma \beta=\left.V_{+}\right|_{\Gamma}-\left.V_{-}\right|_{\Gamma}=0, \quad \gamma(\alpha+i \lambda \beta)=\left.\frac{\partial V_{-}}{\partial \nu}\right|_{\Gamma}-\left.\frac{\partial V_{+}}{\partial \nu}\right|_{\Gamma}=0 .
$$

Hence $\alpha=0$ and $\beta=0$.

Since $\delta \in \bar{X}(D)$, there exists Herglotz wave functions $h_{j}$, such that $h_{j} \rightarrow \delta$. Notice that $h_{j}$ satisfies the Helmholtz equation $\Delta h_{j}+k^{2} h_{j}=0$ in $R^{3}$. We set $\tilde{W}=\delta$, then $(W, \tilde{W})$ satisfies the condition of being an eigenvalue in the sense of definition 4.1 from the fact that

$$
\Delta W+k^{2} W=-|q| \delta, \quad \text { in } R^{3} \backslash \bar{\Gamma} .
$$

From the assumption we conclude that $(W, \tilde{W})$ has to vanish and therefor also $\delta$. Thus $\mathcal{H}^{*}$ is injective and the lemma is proven.

Lemma 6. If $k^{2}$ is not the interior transmission eigenvalue of the domain $D$, then the operator $\mathcal{F}: H \rightarrow L^{2}(S)$ is injective and has dense range.

Proof. Note the fact that $\mathcal{F}(\delta, \alpha, \beta)(\hat{x})$ is the far field pattern of $P(\delta, \alpha, \beta)(x)$ for $(\sqrt{|q|} \delta, \alpha, \beta) \in H$. The injection of the operator $\mathcal{F}$ can be obtained as the derivation of $\mathcal{H}$.

Note that the transpose operator $\mathcal{F}^{*}: L^{2}(S) \rightarrow H^{*}$ is given by

$$
\left(\gamma^{-1} \mathcal{F}^{*} \sigma\right)(y)= \begin{cases}\sqrt{|q|} v_{\sigma} & \text { on } D, \\ v_{\sigma}^{+} & \text {on } \Gamma, \\ \frac{\partial v_{\sigma}^{-}}{\partial \nu(y)} & \text { on } \Gamma .\end{cases}
$$

It is enough to show that $\mathcal{F}^{*}$ is injective. But $\mathcal{F}^{*} \sigma=(0,0,0)$ implies that there exists a Herglotz wave function with $\left.v_{\sigma}\right|_{\Gamma}=0$ and $\left.\frac{\partial v_{\sigma}}{\partial \nu}\right|_{\Gamma}=0$ (note that the limit of $v_{\sigma}$ and its normal derivative from both sides of the crack is the same). From the analyticity of $v_{\sigma}$, we have that $v_{\sigma}=0$ in $R^{3}$ and therefor $\sigma=0$. This proves the lemma.

Remark. We can not prove the injectivity of $\mathcal{H}^{*}$ and $\mathcal{F}$ in the space $L^{2}(D) \times$ $\tilde{H}^{-1 / 2}(\Gamma) \times \tilde{H}^{1 / 2}(\Gamma)$, this is why we choose the space $X_{q}(D)$ instead of $L^{2}(D)$, furthermore, the problem (4.1) is well-posedness for $\tilde{h} \in X_{q}(D)$. 
The next lemma helps us to explain why we select $Q^{\infty}$ which is the far field pattern of the potential $Q(x)$ defined by (4.4) as the right hand side of the far field equation.

Lemma 7. For any open bounded domain $\Sigma$ with smooth boundary and for any smooth non intersecting open arc $L$ without cups such that $\bar{L} \cap \bar{\Sigma}=\emptyset$, let functions $\delta_{\Sigma} \in \bar{X}(\Sigma), \alpha_{L} \in \tilde{H}^{-1 / 2}(L)$ and $\beta_{L} \in \tilde{H}^{1 / 2}(L)$, then $Q^{\infty}(\hat{x}) \in L^{2}(S)$ defined by

$$
\begin{aligned}
Q^{\infty}(\hat{x})= & \gamma \int_{\Sigma}|q(y)| \delta_{\Sigma}(y) e^{-i k \hat{x} \cdot y} d s(y)+\gamma \int_{L} \alpha_{L}(y) e^{-i k \hat{x} \cdot y} d s(y) \\
& +\gamma \int_{L} \beta_{L}(y) \frac{\partial e^{-i k \hat{x} \cdot y}}{\partial \nu(y)} d s(y), \quad \hat{x} \in S
\end{aligned}
$$

is in the range of $R(\mathcal{F})$ if and only if $L \subset \Gamma$ and $\Sigma \cap D \neq \emptyset$.

Proof. First assume that $L \subset \Gamma$ and $\Sigma \cap D \neq \emptyset$. Since $q=0$ outside $D$, the first integral term indeed integrates on $\Sigma \cap D$. Note that $\bar{X}(\Sigma \cap D) \subset \bar{X}(D)$ together with $\tilde{H}^{ \pm 1 / 2}(L) \subset \tilde{H}^{ \pm 1 / 2}(\Gamma)$, it follows from the definition of $\mathcal{F}$ that $Q^{\infty} \in R(\mathcal{F})$.

Now we assume that at least one of the conditions: $L \subset \Gamma, \Sigma \cap D \neq \emptyset$ is not satisfied. There are three situations, here we just prove the lemma under the assumption $L \subset \Gamma, \Sigma \cap D=\emptyset$. In such a case, and on the contrary if $Q^{\infty} \in R(\mathcal{F})$, i.e. there exists $\delta \in \bar{X}(D), \alpha \in \tilde{H}^{-1 / 2}(\Gamma)$ and $\beta \in \tilde{H}^{1 / 2}(\Gamma)$ such that

$$
\begin{aligned}
Q^{\infty}(\hat{x})= & \gamma \int_{D}|q(y)| \delta(y) e^{-i k \hat{x} \cdot y} d s(y)+\gamma \int_{\Gamma} \alpha(y) e^{-i k \hat{x} \cdot y} d s(y) \\
& +\gamma \int_{\Gamma} \beta(y) \frac{\partial e^{-i k \hat{x} \cdot y}}{\partial \nu(y)} d s(y), \quad \hat{x} \in S .
\end{aligned}
$$

Then by Rellich's lemma and unique continuation principle we have that the two potentials

$$
\begin{aligned}
& Q(x)=\int_{L} \alpha_{L}(y) \Phi(x, y) d s(y)+\int_{L} \beta_{L}(y) \frac{\partial \Phi(x, y)}{\partial \nu(y)} d s(y) \\
& P(x)=\int_{D}|q(y)| \delta(y) \Phi(x, y) d s(y)+\int_{\Gamma} \alpha(y) \Phi(x, y) d s(y)+\int_{\Gamma} \beta(y) \frac{\partial \Phi(x, y)}{\partial \nu(y)} d s(y)
\end{aligned}
$$

coincide in $R^{3} \backslash(\bar{D} \cup \bar{\Sigma} \cup \bar{\Gamma})$.

One can see that $Q$ is analytic in $R^{3} \backslash \bar{\Gamma}$, however $P$ has singularity on the boundary $\partial D$ which is a contradiction and we prove the lemma.

Remark. For the case of $L \not \subset \Gamma$ and $\Sigma \cap D \neq \emptyset$ or $L \not \subset \Gamma$ and $\Sigma \cap D=\emptyset$, we can also derive a contradiction by a similar discussion.

At this time, we can recover the mixed-type scatterer which consists of a crack and a penetrable inhomogeneous medium by the linear sampling method via solving the far field equation (4.3). The result for the reconstruction of the combined scatterer is stated as following. 
Theorem 5. Assume that $\Gamma$ is an oriented nonintersecting piecewise smooth arc without cusps, $\partial D$ is smooth and $k^{2}$ is not the interior transmission eigenvalue of the domain $D$. Then if $F$ which is defined by (4.2) is the far field operator corresponding to the scattering problem (1.1) and (1.2), the following are true:

(1) If $L \subset \Gamma, \Sigma \cap D \neq \emptyset$, then for every $\epsilon>0$ there exists a solution $g_{L, \Sigma}^{\epsilon} \in L^{2}(S)$ satisfying

$$
\left\|F g_{L, \Sigma}^{\epsilon}+Q^{\infty}\right\|_{L^{2}(S)}<\epsilon .
$$

(2) If $L \not \subset \Gamma$ or $\Sigma \cap D=\emptyset$, then for every $\epsilon>0$ and $\varepsilon>0$ there exists a function $g_{L, \Sigma}^{\epsilon, \varepsilon} \in L^{2}(S)$ such that

$$
\left\|F g_{L, \Sigma}^{\epsilon, \varepsilon}+Q^{\infty}\right\|_{L^{2}(S)}<\epsilon+\varepsilon, \quad \lim _{\varepsilon \rightarrow 0}\left\|g_{L, \Sigma}^{\epsilon, \varepsilon}\right\|_{L^{2}(S)}=\infty .
$$

Proof. If $L \subset \Gamma, \Sigma \cap D \neq \emptyset$, then by Lemma 4.3, the corresponding $\left(\sqrt{|q|} \delta_{\Sigma \cap D}\right.$, $\left.\alpha_{L}, \beta_{L}\right)$ is in $H$. Since $M\left(\delta_{\Sigma \cap D}, \alpha_{L}, \beta_{L}\right) \in H^{*}$, then from Lemma 4.1, for every $\epsilon_{0}>0$ there exists a function $g_{L, \Sigma}^{\epsilon_{0}} \in L^{2}(S)$ such that

$$
\left\|M\left(\alpha_{L}, \beta_{L}, \delta_{\Sigma}\right)-\mathcal{H} g_{L, \Sigma}^{\epsilon_{0}}\right\|_{H^{*}}<\epsilon_{0} .
$$

Whence from the continuity of $M^{-1}$

$$
\left\|\left(\alpha_{L}, \beta_{L}, \delta_{\Sigma}\right)-M^{-1} \mathcal{H} g_{L, \Sigma}^{\epsilon_{0}}\right\|_{H}<C_{1} \epsilon_{0}
$$

with a positive constant $C_{1}$. Finally, the formula (4.6), the continuity of $\mathcal{F}$ and the fact that $\mathcal{F}\left(\alpha_{L}, \beta_{L}, \delta_{\Sigma}\right)=Q^{\infty}$ imply that

$$
\left\|F g_{L, \Sigma}^{\epsilon}+Q^{\infty}\right\|_{L^{2}(S)}<C_{2} \epsilon_{0} .
$$

Then (4.8) is confirmed with $\epsilon=C_{2} \epsilon_{0}$.

Next, we assume that $L \not \subset \Gamma$ or $\Sigma \cap D=\emptyset$. In this case, by Lemma $4.3 Q^{\infty}$ is not in the range of $\mathcal{F}$. But from lemma 4.2 we know that the operator $\mathcal{F}$ is compact and injective with dense range in $L^{2}(S)$. Hence for every $\varepsilon>0$ we can construct a unique Tikhonov regularized solution $\left(\sqrt{|q|} \delta_{\Sigma}^{\rho}, \alpha_{L}^{\rho}, \beta_{L}^{\rho}\right) \in H$ of equation $\mathcal{F}(\alpha, \beta, \delta)=Q^{\infty}$, such that

$$
\left\|\mathcal{F}\left(\delta_{\Sigma}^{\rho}, \alpha_{L}^{\rho}, \beta_{L}^{\rho}\right)-Q^{\infty}\right\|_{L^{2}(S)}<\varepsilon,
$$

where $\rho$ is the regularization parameter (chosen by a regular regularization strategy, e.g. the Morozov discrepancy principle). Then we have $\|\left(\sqrt{|q|} \delta_{\Sigma}^{\rho}\right.$, $\left.\alpha_{L}^{\rho}, \beta_{L}^{\rho}\right) \|_{H} \rightarrow \infty$ as $\rho \rightarrow 0$. Now the above considerations for $\left(\delta_{\Sigma}, \alpha_{L}, \beta_{L}\right)$ can be applied to $\left(\delta_{\Sigma}^{\rho}, \alpha_{L}^{\rho}, \beta_{L}^{\rho},\right)$. Hence for $\epsilon_{1}>0$ sufficient small there exists $g_{L, \Sigma}^{\epsilon_{1}, \rho}$ such that

$$
\left\|M\left(\delta_{\Sigma}^{\rho}, \alpha_{L}^{\rho}, \beta_{L}^{\rho}\right)-\mathcal{H} g_{L, \Sigma}^{\epsilon_{1}, \rho}\right\|_{H^{*}}<\epsilon_{1}
$$


and

$$
\left\|\left(\sqrt{|q|} \delta_{\Sigma}^{\rho}, \alpha_{L}^{\rho}, \beta_{L}^{\rho}\right)-M^{-1} \mathcal{H} g_{L, \Sigma}^{\epsilon_{1}, \rho}\right\|_{H}<C_{1} \epsilon_{1} .
$$

Combining (4.9) and (4.10) we obtain that for every $\epsilon>0$ and $\varepsilon>0$ there exists a $g_{L, \Sigma}^{\epsilon, \rho} \in L^{2}(S)$ such that

$$
\begin{aligned}
\left\|F g_{L, \Sigma}^{\epsilon, \rho}+Q^{\infty}\right\|_{L^{2}(S)}= & \left\|\mathcal{F} M^{-1} \mathcal{H} g_{L, \Sigma}^{\epsilon, \rho}-Q^{\infty}\right\|_{L^{2}(S)} \\
\leq & \left\|\mathcal{F} M^{-1} \mathcal{H} g_{L, \Sigma}^{\epsilon, \rho}-\mathcal{F}\left(\delta_{\Sigma}^{\rho}, \alpha_{L}^{\rho}, \beta_{L}^{\rho}\right)\right\|_{L^{2}(S)} \\
& +\left\|\mathcal{F}\left(\delta_{\Sigma}^{\rho}, \alpha_{L}^{\rho}, \beta_{L}^{\rho}\right)-Q^{\infty}\right\|_{L^{2}(S)}<\epsilon+\varepsilon
\end{aligned}
$$

with $\epsilon=C_{1} \epsilon_{1}$. Since $\lim _{\varepsilon \rightarrow 0} \rho(\varepsilon)=0$ we have that $\lim _{\varepsilon \rightarrow 0} \|\left(\sqrt{|q|} \alpha_{L}^{\rho}\right.$, $\left.\beta_{L}^{\rho}, \delta_{\Sigma}^{\rho}\right) \|_{H} \rightarrow \infty$. From (4.10) and the boundness of $M^{-1}$ we have that $\lim _{\varepsilon \rightarrow 0}\left\|\mathcal{H} g_{L, \Sigma}^{\epsilon, \varepsilon}\right\|_{H^{*}} \rightarrow \infty$. By the definition of the operator $\mathcal{H}$ we have that

$$
\lim _{\varepsilon \rightarrow 0}\left\|g_{L, \Sigma}^{\epsilon, \varepsilon}\right\|_{L^{2}(S)} \rightarrow \infty
$$

Then we complete the proof of this theorem.

\section{References}

[1] X. Antoine, C. Chniti and K. Ramdani. On the numerical approximation of highfrequency acoustic multiple scattering problems by circular cylinders. J. Comput. Phys., 227:1754-1771, 2008. http://dx.doi.org/10.1016/j.jcp.2007.09.030.

[2] T. Arens and A. Lechleiter. The linear sampling method revisited. J. Integral Equations Appl., 21:179-202, 2009.

http://dx.doi.org/10.1216/JIE-2009-21-2-179.

[3] F. Cakoni and D. Colton. On the mathematic basis of the linear sampling method. Georg. Math. J., 10:411-425, 2003.

[4] F. Cakoni and D. Colton. Qualitative Method in Inverse Scattering Theory. Springer, Berlin, 2006.

[5] D. P. Challa, G. Hu and M. Sini. Multiple scattering of electromagnetic waves by a finite number of point-like obstacles. WIAS Preprint, No. 1745, 2012.

[6] D.P. Challa and M. Sini. Inverse scattering by point-like scatterers in the foldy regime. Inverse Problems, 28:125006, 2012. http://dx.doi.org/10.1088/0266-5611/28/12/125006.

[7] J. Cheng, J.J. Liu, G. Nakamura and S.Z. Wang. Recovery of multiple obstacles by probe method. Quart. Appl. Math., 67:221-247, 2009. http://dx.doi.org/10.1090/S0033-569X-09-01101-0.

[8] D. Colton, H. Haddar and M. Piana. The linear sampling method in inverse electromagnetic scattering theory. Inverse Problems, 19:S105-S137, 2002. http://dx.doi.org/10.1088/0266-5611/19/6/057.

[9] D. Colton and A. Kirsch. A simple method for solving inverse scattering problems in the resonance region. Inverse Problems, 12:383-393, 1996.

http://dx.doi.org/10.1088/0266-5611/12/4/003.

[10] D. Colton and R. Kress. Inverse Acoustic and Electromagnetic Scattering Theory (2nd Edition). Springer, Berlin, 1998. 
[11] M. Ganesh and S.C. Hawkins. Simulation of acoustic scattering by multiple obstacles in three dimensions. ANZIAM J., 50:C31-C45, 2008.

[12] M. Ganesh and S.C. Hawkins. An efficient algorithm for simulating scattering by a large number of two dimensional particles. ANZIAM J., 52:C139-C155, 2011.

[13] N.I. Grinberg. Obstacle visualization via the factorization method for the mixed boundary value problem. Inverse Problems, 18:1687-1704, 2002.

http://dx.doi.org/10.1088/0266-5611/18/6/317.

[14] N.I. Grinberg and A. Kirsch. The factorization method for obstacles with a-priori separated sound-soft and sound-hard parts. Math. Comput. Simul., 66:267-279, 2004. http://dx.doi.org/10.1016/j.matcom.2004.02.011.

[15] J. Guo, L.L. Fan and G.Z. Yan. The boundary integral method for the Helmholtz equation with cracks inside a bounded domain. Acta Math. Sci., 35:539-551, 2015. http://dx.doi.org/10.1016/S0252-9602(15)30002-3.

[16] F.B. Hassen, J.J. Liu and R. Potthast. On source analysis by wave splitting with applications in inverse scattering of multiple obstacles. J. Comput. Math., 25:266-281, 2007.

[17] G. Hu, M. Andrea and M. Sini. Direct and inverse acoustic scattering by a collection of extended and point-like scatterers. Multiscale Model. Simul., 12:996-1027, 2014. http://dx.doi.org/10.1137/130932107.

[18] G. Hu and M. Sini. Elastic scattering by finitely many point-like obstacles. J. Math. Phys., 54:042901, 2013. http://dx.doi.org/10.1063/1.4799145.

[19] K. Huang and P. Li. A two-scale multiple scattering problem. Multiscale Model. Simul., 8:1511-1534, 2010. http://dx.doi.org/10.1137/090771090.

[20] K. Huang, K. Solna and H. Zhao. Generalized Foldy-Lax formulation. J. Comput. Phys., 229:4544-4553, 2010. http://dx.doi.org/10.1016/j.jcp.2010.02.021.

[21] A. Kirsch and X. Liu. Direct and inverse acoustic scattering by a mixed-type scatterer. Inverse Problems, 29:065005, 2013. http://dx.doi.org/10.1088/0266-5611/29/6/065005.

[22] J.J. Liu, P.A. Krutitskii and M. Sini. Numerical solution of the scattering problem for acoustic waves by a two-sided crack in 2-dimensional space. J. Comput. Math., 29:141-166, 2011.

[23] J.J. Liu and M. Sini. Reconstruction of cracks of different types from far-field measurements. Math. Methods Appl. Sci., 33:950-973, 2010.

[24] X. Liu and B. Zhang. Inverse scattering by an inhomogeneous penetrable obstacle in a piecewise homogeneous medium. Acta Math. Sci., 32:1281-1297, 2012. http://dx.doi.org/10.1016/S0252-9602(12)60099-X.

[25] P.A. Martin. Multiple Scattering. Encyclopedia Math. Appl. Cambridge University Press, Cambridge, 2006.

[26] G. Uhlmann. Electrical impedance tomography and Calderóns problem. Inverse Problems, 25:123001, 2009. http://dx.doi.org/10.1088/0266-5611/25/12/123011.

[27] J. Yang, B. Zhang and H. Zhang. Uniqueness in inverse acoustic and electromagnetic scattering by penetrable obstacles. Math. AP, 2013. arXiv:1305.0917 